\title{
(6) OPEN ACCESS \\ Vaccination against influenza in patients with rheumatoid arthritis: the effect of rituximab on the humoral response
}

\author{
S Oren, ${ }^{1}$ M Mandelboim, ${ }^{2}$ Y Braun-Moscovici, ${ }^{3}$ D Paran, ${ }^{1}$ J Ablin,,${ }^{1,4}$ I Litinsky, ${ }^{1}$ \\ D Comaneshter, ${ }^{5}$ D Levartovsky, ${ }^{1}$ E Mendelson, ${ }^{2} \mathrm{R}$ Azar, ${ }^{2}$ I Wigler, ${ }^{1}$ A Balbir-Gurman, ${ }^{3}$ \\ D Caspi, 0 Elkayam ${ }^{1,4}$
}

${ }^{1}$ Department of Rheumatology, Tel Aviv Sourasky Medical Centre, Sackler Faculty of Medicine, Tel Aviv University, Tel Aviv, Israel; ${ }^{2}$ Central Virology Laboratory, Ministry of Health, Chaim Sheba Medical Centre, Tel Hashomer, Israel; Tel Aviv Medical Centre, Sackler Faculty of Medicine, Tel Aviv University, Tel Aviv, Israel; ${ }^{3} \mathrm{~B}$ Shine Department of Rheumatology, Rambam Health Care Centre, The Ruth and Bruce Rapaport School of Medicine, Technion, Israel Institute of Technology, Haifa, Israel; ${ }^{4}$ Department of Internal Medicine "F", Tel Aviv Sourasky Medical Centre, Sackler Faculty of Medicine, Tel Aviv University, Tel Aviv, Israel;

${ }^{5}$ Statistic Unit, Tel Aviv

Sourasky Medical Centre, Tel Aviv, Israel

Correspondence to: Dr 0 Elkayam, Department of Rheumatology, Tel Aviv Sourasky Medical Centre, 6 Weizmann Street, Tel Aviv 64239, Israel;

oribe14@netvision.net.il

Accepted 13 October 2007

Published Online First

4 December 2007

\section{ABSTRACT}

Objective: To assess the effect of rituximab on the efficacy and safety of influenza virus vaccine in patients with rheumatoid arthritis (RA).

Methods: The study group comprised patients with RA treated with conventional disease-modifying drugs with or without rituximab. Split-virion inactivated vaccine containing $15 \mu \mathrm{g}$ haemagglutinin/dose of B/Shanghai/361/02 (SHAN), A/New Caledonian/20/99 (NC) (H1N1) and A/ California/7/04 (CAL) (H3N2) was used. Disease activity was assessed by the number of tender and swollen joints, duration of morning stiffness and evaluation of pain on the day of vaccination and 4 weeks later. CD19-positive cell levels were assessed in rituximab-treated patients. Haemagglutination inhibition (HI) antibodies were tested and response was defined as a greater than fourfold rise 4 weeks after vaccination or seroconversion in patients with a non-protective baseline level of antibodies $(<1 /$ 40). Geometric mean titres (GMT) were calculated in all subjects.

Results: The participants were divided into three groups: RA ( $n=29$, aged 64 (12) years), rituximab-treated RA ( $n=14$, aged 53 (15) years) and healthy controls ( $n=21$, aged 58 (15) years). All baseline protective levels of $\mathrm{HI}$ antibodies and GMT were similar. Four weeks after vaccination, there was a significant increase in GMT for NC and CAL antigens in all subjects, but not for the SHAN antigen in the rituximab group. In rituximab-treated patients, the percentage of responders was low for all three antigens tested, achieving statistical significance for the CAL antigen. Measures of disease activity remained unchanged.

Conclusion: Influenza virus vaccine generated a humoral response in all study patients with RA and controls. Although the response was significantly lower among rituximab-treated patients, treatment with rituximab does not preclude administration of vaccination against influenza.

Infection is one of the leading causes of morbidity and mortality in patients with rheumatoid arthritis (RA), ${ }^{12}$ who have nearly twice the rate of infection compared with matched non-RA controls. ${ }^{3}$ This might be related to the disease itself through either altered immunological function or due to other factors, such as immobility, skin defects, Felty's syndrome and more. Alternatively, treatment with corticosteroids and other immunosuppressive drugs may also predispose patients with RA to the development of severe infections. ${ }^{4}$
Vaccination against influenza is currently recommended to patients who have chronic diseases, including RA. ${ }^{5}$ We and others ${ }^{67}$ have shown that vaccination against influenza is safe and that it induces a satisfactory humoral response, although one that is lower than in healthy controls. The humoral response of patients with RA to vaccination against influenza does not seem to be affected by the use of prednisone, disease modifying antirheumatic drugs (DMARDs) or tumour necrosis factor $\alpha$ blockers. ${ }^{67}$

Results of recent clinical studies ${ }^{8}$ using the B-celldepleting monoclonal antibody rituximab indicate that B-cell-targeted treatment promises to be a major advance in the treatment of RA. B-lymphocyte depletion with rituximab, either alone or in combination with cyclophosphamide or methotrexate (MTX), has been shown to have efficacy in patients with RA who experience incomplete response to MTX.

Rituximab is a genetically engineered chimeric anti-CD20 monoclonal antibody that selectively targets CD20+ B cells and induces a transient depletion of the CD20+ mature B-cell subpopulation. Although the levels of pre-existing antibody levels against tetanus and pneumococcal polysaccharide were shown to be unaffected by a single course of rituximab, ${ }^{9}$ its effect on the immune response to active immunisation in patients with RA has not yet been investigated. The purpose of our study was to evaluate the effect of rituximab on the immunogenicity of vaccination against influenza in patients with RA and to assess safety in these patients.

\section{PATIENTS AND METHODS}

\section{Subjects}

Forty-three consecutive outpatients routinely treated at two departments of rheumatology who fulfilled the American College of Rheumatology criteria for $\mathrm{RA}^{10}$ and 21 healthy hospital personnel matched for age and gender participated in the study. Fourteen of the 43 patients with RA had been treated with rituximab (rituximab group) during the previous 18 months (seven of them during the past 6 months) according to the standard protocol used in RA-that is, two infusions of $1000 \mathrm{mg}$ each along with $100 \mathrm{mg}$ methylprednisolone intravenously with background weekly MTX. The remaining 29 patients with RA (non-rituximab) were receiving different 
DMARDs excluding tumour necrosis factor $\alpha$ blockers or other biological agents. Patients were required to be on stable drug treatment for the 3 months preceding the vaccination. The vaccinations consisted of $0.5 \mathrm{ml}$ split virion inactivated vaccine (Vaxigrip, Promedico) containing a $15 \mu \mathrm{g}$ haemagglutinin (HA) dose of A/California /7/04 (CAL) (H3N2), B/Shanghai /361/02 (SHAN) and A/New Caledonian/20/99 (NC) (H1N1), administered intramuscularly. Exclusion criteria were pregnancy, a history of past vaccination allergy, a known allergy to egg products, hyposplenism, and active RA necessitating a recent change in the drug regimen.

\section{Clinical assessment}

Each subject gave a complete history including drug use and underwent a physical examination before vaccination. Clinical assessment on the day of vaccination and 4 weeks later included the following: duration of morning stiffness (in minutes); evaluation of daytime and nocturnal pain using a visual analogue scale of $10 \mathrm{~cm}$, and count of the number of tender and swollen joints (28 joint counts).

Laboratory assessment of disease activity included erythrocyte sedimentation rate (ESR) and C-reactive protein (CRP) on the day of the vaccination and 4 weeks later. A complete blood count was performed for all treated patients, with assessment of total lymphocyte count and the subpopulations, including CD5, CD19 and CD20, assessed as frequency and absolute numbers.

\section{Haemagglutination inhibition test}

The immunogenicity of the vaccine was tested by the haemagglutination inhibition (HI) test. Influenza virus has two important surface glycoproteins, HA and neuraminidase. Antigenic classification and subtyping of influenza viruses are based on these two glycoproteins. HA has a key role in virus cell entry by binding to cell surface receptors, which are also found on red blood cells of certain species. Binding to red cells results in haemagglutination, which can be observed as a carpet of agglutinated red cells at the bottom of a tube or microtitre well. In the HI test, antibodies directed against the viral HAs block the virus from binding to the blood cells and thus inhibit the haemagglutination reaction.

The pre- and postimmunisation HI antibodies were tested at the Central Virology Laboratory of the Israeli Ministry of Health using the HI test according to a standard WHO procedure. ${ }^{11}$ Serum samples were separated, code labelled, and stored at $-20^{\circ} \mathrm{C}$ until tested. They were treated with receptordestroying enzyme cholera filtrate to remove non-specific inhibitors, and with Turkey red blood cells to remove nonspecific agglutinins. The treated serum samples were tested by an $\mathrm{HI}$ test against the three antigens included in the vaccine, CAL, SHAN and NC. The working dilution (test dose) of each antigen contained four HA units in $25 \mu \mathrm{l}$ of antigen. Test doses were diluted in phosphate-buffered saline and added to serial dilution of antiserum. The HA inhibition titre was determined as the highest dilution of serum that completely inhibited haemagglutination of red blood cells.

The titre of an antiserum not showing any inhibition was recorded as $<10$. Humoral response was defined as either a fourfold or more rise in titre, or a rise from a non-protective baseline level of $<1 / 40$ to $\geqslant 1 / 40$ in $\mathrm{HI}$ antibodies 4 weeks after vaccination. ${ }^{12} 13$ Geometric mean titres of antibodies were calculated to assess the immunity of the whole group.

\section{Outcomes of the study}

The primary outcome was the percentage of patients from the three groups (RA treated with rituximab, RA treated with DMARDs alone and controls) showing a humoral response to each of the three serotypes. Secondary outcomes included predictors of immunogenicity in patients with RA and the safety of the vaccine in these patients.

Appropriate informed consent was obtained from all patients, and the clinical research was conducted in accordance with guidelines for human experimentation specified by the Tel Aviv Sourasky Medical Centre and the Rambam Health Care Centre. The study was approved by the ethics committee of both medical centres.

\section{Statistical methods}

Non-parametric tests were used for the analysis since most variables were not normally distributed (based on the ShapiroWilk test). In addition, parametric tests were performed for the log transformation of the variables.

Associations between the response to vaccination and patient group and the drug use were examined using the $\chi^{2}$ and the Fisher exact tests.

Patients with positive reactions to vaccination were compared with those who did not react with respect to clinical measures-use of drugs at baseline, change in disease indices (number of tender and swollen joints, morning stiffness, pain intensity), ESR, CRP, etc-using the Mann-Whitney U test and the $t$ test for independent samples.

Change in drug use was evaluated by the McNemar test, change in the number of drugs by the Wilcoxon non-parametric test, and change in drug dosage by paired $t$ tests. A binomial logistic regression model was constructed to assess the importance of the different variables relative to the immunogenicity response.

Statistical analysis was carried out using the SPSS system for Windows, release 14.0.

\section{RESULTS}

\section{Characteristics of patients and control subjects}

The three groups were statistically similar in age and gender and comprised mostly women (table 1). The mean disease duration was 15.5 years for the patients with RA and 16.5 years in the rituximab group. At the time of vaccination, all patients with RA were being treated with at least one DMARD (table 2). Most $(76 \%)$ of the non-rituximab RA subjects and $86 \%$ of the

Table 1 Clinical and demographic characteristics of patients with rheumatoid arthritis (RA) with and without rituximab treatment and of healthy controls

\begin{tabular}{lllll}
\hline Characteristics & $\begin{array}{l}\text { RA } \\
(\mathbf{n}=\mathbf{2 9})\end{array}$ & $\begin{array}{l}\text { RA + rituximab } \\
(\mathbf{n}=\mathbf{1 4})\end{array}$ & $\begin{array}{l}\text { Controls } \\
(\mathbf{n}=\mathbf{2 1})\end{array}$ & $\mathbf{p}$ Value \\
\hline Age (years), mean (SD) & $64(12)$ & $53(15)$ & $58(15)$ & $\mathrm{NS}$ \\
Sex (F:M), No (\%) & $22(76): 7(24)$ & $13(93): 1(7)$ & $14(67): 7(33)$ & $\mathrm{NS}$ \\
RA duration (years), mean (SD) & $15.5(11)$ & $16.5(10)$ & - & NS \\
\hline
\end{tabular}


Table 2 Drugs used by patients with rheumatoid arthritis (RA) at the time of influenza virus vaccination

\begin{tabular}{lll}
\hline Drug & $\begin{array}{l}\text { RA } \\
(\mathbf{n}=\mathbf{2 9})\end{array}$ & $\begin{array}{l}\text { RA + rituximab } \\
(\mathbf{n}=14)\end{array}$ \\
\hline MTX & & \\
$\quad$ Patients, No (\%) & $22(76)$ & $12(86)$ \\
$\quad$ Dose (mg/week), mean (SD) & $12.7(4.3)$ & $14.6(3.8)$ \\
Prednisone & & \\
$\quad$ Patients, No (\%) & $8(28)$ & $7(50)$ \\
$\quad$ Dose (mg/day), mean (SD) & $6.3(2.2)$ & $9.6(0.9)^{*}$ \\
Hydroxychloroquine, No (\%) & $10(34)$ & $3(21)$ \\
Sulfasalazine, No (\%) & $2(7)$ & $1(7)$ \\
Leflunomide, No (\%) & $2(7)$ & $0(0)$ \\
Azathioprine, No (\%) & $1(3)$ & $0(0)$ \\
Minocycline, No (\%) & $1(3)$ & $0(0)$ \\
Gold, No (\%) & $1(3)$ & $0(0)$ \\
NSAIDs, No (\%) & $6(21)$ & $2(14)$ \\
\hline * $p=0.003$. & \\
MTX, methotrexate; NSAIDs, non-steroidal anti-inflammatory drugs.
\end{tabular}

rituximab RA subjects were being treated with MTX at a mean dose of 12.7 and $14.6 \mathrm{mg} /$ week, respectively. A significantly higher proportion of patients in the rituximab group was treated with prednisone ( $50 \%$ vs $28 \%$ non-rituximab, $p=0.003$ ) At the time of vaccination, patients in the rituximab group were profoundly B-cell depleted, with a mean (SD) level of 14.2 (22.8) CD19+ B cells, $0.98 \%$ of total lymphocytes (range 0-80 $\mu \mathrm{l}$ ). Four weeks after vaccination, the mean level of $\mathrm{CD} 19+\mathrm{B}$ cells was 22 , $1.6 \%$ of total lymphocytes (range $0-50 \mu \mathrm{l}$ ).

\section{Effect of vaccination against influenza on disease activity}

Vaccination against influenza was not associated with a significant worsening of any clinical or laboratory indices of disease activity in both groups of rheumatoid patients (table 3). A few subjects reported mild adverse events after vaccination: two rheumatoid patients in the rituximab group and none of the controls developed symptoms of mild upper respiratory tract infection within 4 weeks following vaccination.

\section{Immunogenicity of influenza vaccine}

Pre-vaccination HI antibody levels-that is, as a result of previous infection or vaccination, did not differ significantly between patients with RA and controls. Four weeks after vaccination, all study participants in the control groups had significant increases in their geometric mean titres of $\mathrm{HI}$ antibody against each of the antigens tested, suggesting a satisfactory humoral response on the part of the whole group. The rituximab group showed a significant rise for $\mathrm{NC}$ and CAL antigens but not for SHAN (fig 1).

\section{Individual responses of rheumatoid patients and controls to vaccination against influenza}

Although patients with RA and controls responded to most of the antigens, the vaccine did not appear to be uniformly immunogenic among all the patients. Levels of $\mathrm{HI}$ antibodies $<1 / 40$ are considered to be non-protective. A satisfactory humoral response was defined as a fourfold or more rise in $\mathrm{HI}$ antibodies 4 weeks after vaccination in patients with baseline HI antibody levels $>1 / 40$, or a rise of HI levels to $\geqslant 1 / 40$ in patients with non-protective baseline levels of $<1 / 40$. When this definition was used, the proportion of responders to the CAL antigen was significantly lower in the rituximab group than in the non-rituximab group ( $21 \%$ vs $67 \%$, respectively;
Table 3 Effects of influenza virus vaccination on disease activity in all 43 study patients with rheumatoid arthritis

\begin{tabular}{lccl}
\hline Disease activity measures & $\begin{array}{l}\text { Before } \\
\text { vaccination }\end{array}$ & $\begin{array}{l}\text { After } \\
\text { vaccination }\end{array}$ & p Value \\
\hline Tender joints (n) & $7.1(10)$ & $6.4(8)$ & 0.51 \\
Swollen joints (n) & $2.5(3.5)$ & $2.5(3.7)$ & 0.89 \\
Morning stiffness (min) & $25(56)$ & $33(65)$ & 0.17 \\
Day and night pain (VAS) & $4.3(2.3)$ & $4(3)$ & 0.83 \\
ESR (mm/1st h) & $35(20)$ & $34(19)$ & 0.96 \\
C-reactive protein (mg/l) & $13(14)$ & $10(10)$ & $0.024^{*}$ \\
\hline
\end{tabular}

Values are mean (SD).

ESR, erythrocyte sedimentation rate; VAS, visual analogue score.

$p=0.006$ ). The proportion of responders to the SHAN and NC antigens was similar in the three groups (fig 2). Response to more than one antigen was obtained in $14 \%$ of the rituximab patients, $48 \%$ of the non-rituximab patients and $40 \%$ of the healthy controls $(p=0.53)$. No difference was noted between the three groups in the proportion of responders to all three antigens or to none of them.

\section{Predictors of immunogenicity}

We attempted to identify clinical and/or laboratory indices which might predict a poor response to the influenza virus vaccine. We could not find any association between the humoral response and age, sex, disease duration, swollen and tender joint counts, duration of morning stiffness, level of pain, ESR and CRP levels, the use or dose of prednisone or MTX. The proportion of responders to the CAL antigen was significantly higher in patients treated with a smaller number of DMARDs ( 3.95 vs $7, p=0.02$ ) while no difference was noted for the two other antigens. Multivariate regression analysis did not identify any predictor of immunogenicity. We also could not find any correlation between immunogenicity and the level of CD19+ B cells, $\mathrm{CD}+19$ frequency or the time interval since receiving rituximab in the rituximab-treated patients with RA (table 4), although the very low levels of CD+19 may not allow evaluation of its influence on immunogenicity.

\section{DISCUSSION}

The findings of the current study demonstrated that vaccination against influenza generated a humoral response for two of three antigens tested (NC and CAL but not SHAN) in rheumatoid patients treated with rituximab. The proportion of responders to one of the antigens only (CAL) was significantly lower in the patients treated with rituximab, while the response rate for the other two antigens was similar among all participants. The humoral response was not affected by different clinical and demographic characteristics of RA, or by the level of $B$ cells or the length of time that had passed since receiving rituximab.

The immune responsiveness of patients treated with rituximab has been examined by several studies, most of them performed in patients with lymphoma. Horwitz et al evaluated the ability of 35 patients with lymphoma who were being treated with rituximab and cyclophosphamide to respond to vaccination against tetanus, haemophilus influenza and pneumococcus administered at 6 and 9 months after their last rituximab infusion. ${ }^{14}$ Most of the patients produced protective antibody levels against haemophilus and tetanus but not against pneumococcus. ${ }^{14}$ Others have reported significantly decreased responses to recall antigen, tetanus and polio immunisation and no responses to primary antigen, keyhole 


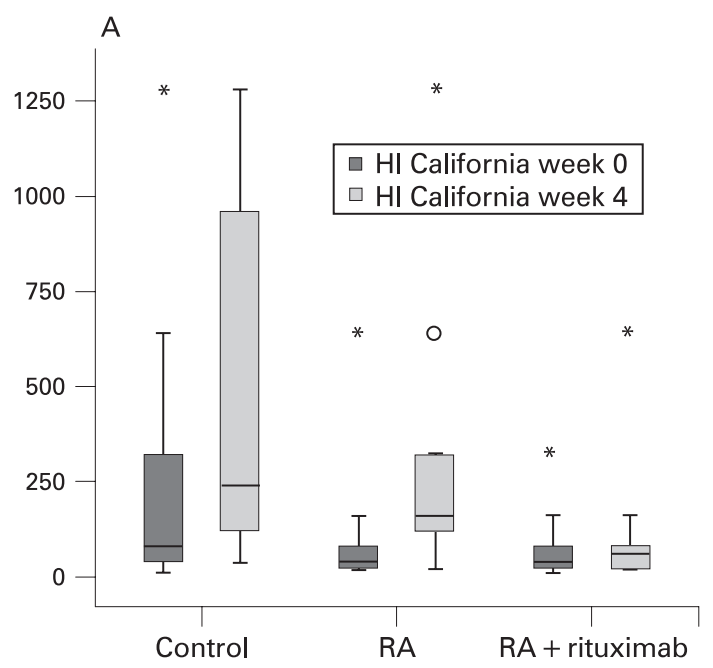

B

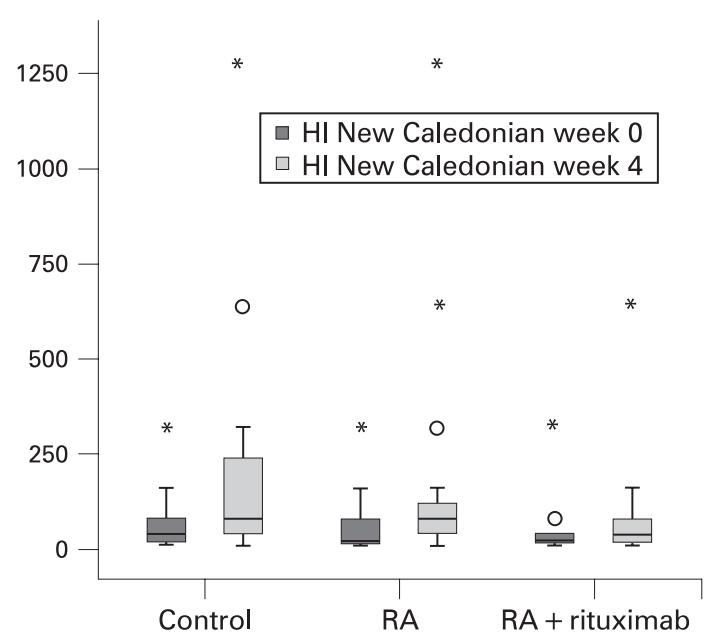

C

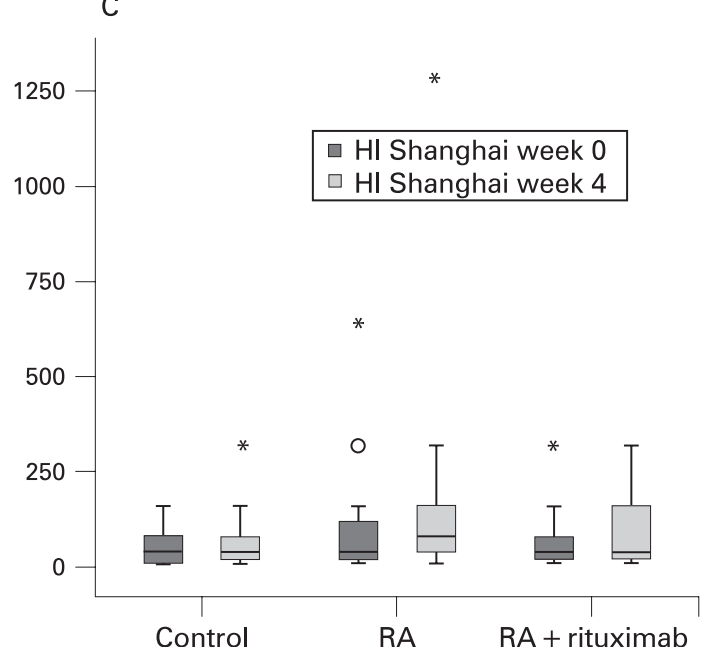

Figure 1 Geometric mean titres of haemagglutination inhibition (HI) antibodies $(\mu \mathrm{g} / \mathrm{ml})$ against influenza antigens in each group of patients with rheumatoid arthritis (RA) and controls before and 4 weeks after influenza virus vaccination. (A) California antigen; (B) New Caledonian; (C) Shanghai.

limpet haemocyanin and hepatitis A immunisation after rituximab monotherapy in 11 patients with lymphoma. ${ }^{15}$ Another study assessed immunisation with neoantigen phiX174 - an established method of assessing in vivo antibody

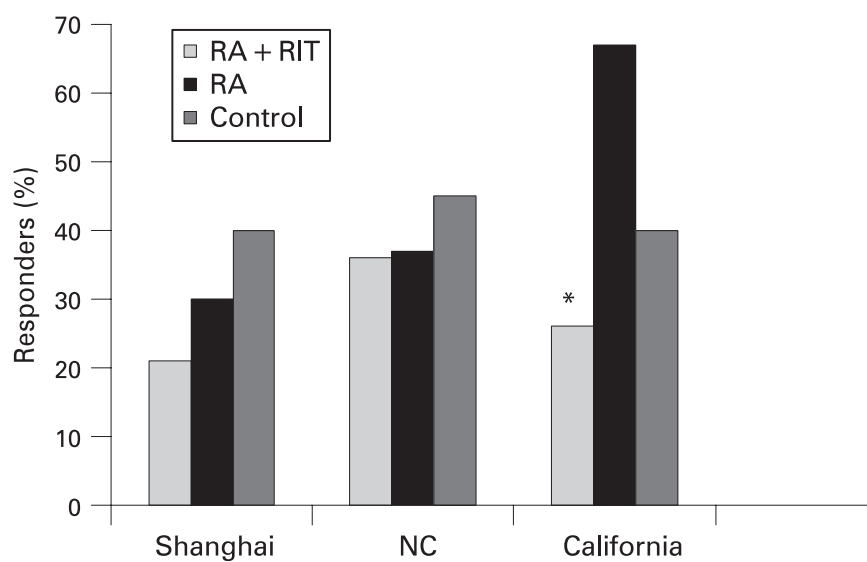

Figure 2 Percentages of responders among both groups of patients with rheumatoid arthritis (RA) and controls. NC, New Caledonian; RIT, rituximab. ${ }^{*} p<0.05$.

response-in patients with chronic renal failure who were treated with rituximab before transplantation. ${ }^{16}$ Rituximab significantly inhibited the antibody response to phiX174 at all time points after both primary and secondary immunisations compared with subjects with chronic renal failure not treated with rituximab $(p<0.001)$ and normal controls $(p<0.001) .{ }^{16}$

Data on the effect of rituximab on immune responsiveness of patients with autoimmune diseases are scarce. Edwards et al demonstrated that despite complete depletion of $\mathrm{B}$ cells in patients with RA treated with rituximab, levels of immunoglobulins as well as anti-tetanus antibody titres remained stable, reflecting the presence of long-living plasma cells. ${ }^{17}$ Although serum levels of rheumatoid factor decreased during treatment with rituximab, no changes in anti-tetanus toxoid or antipneumococcal polysaccharide antibody levels were seen. ${ }^{18}$ In another study," serum levels of IgG anti-tetanus toxoid antibodies and antibodies to pneumococcal capsular polysaccharide did not change significantly in 16 patients with active systemic lupus erythematosus who received rituximab. On the other hand, Vallerskog et al recently investigated how rituximab-induced B-cell depletion affected antibody titres in patients with systemic lupus erythematosus. ${ }^{19}$ Serum levels of $\operatorname{IgG}$ and antibodies against measles and tetanus remained

Table 4 Correlations between immunogenicity and the level of CD19+ $B$ cells and the interval since receiving rituximab

\begin{tabular}{lcccl}
\hline Antigen & No & $\begin{array}{l}\text { Weeks after } \\
\text { rituximab }\end{array}$ & CD19 & \% CD19 \\
\hline B/Shangai & & & & \\
Responders & 3 & $34.3(26)$ & $2.7(2.3)$ & $0.23(0.19)$ \\
Non-responders & 11 & $31.5(2.8)$ & $17.3(25)$ & $1.26(1.56)$ \\
p Value & & 0.875 & 0.391 & 0.357 \\
& & & & \\
A/New Caledonian & & & & \\
Responders & 5 & $32.8(21.4)$ & $18.1(18.4)$ & $0.65(0.35)$ \\
Non-responders & 9 & $31.8(24.4)$ & $12(25.8)$ & $1.11(1.64)$ \\
p Value & & 0.787 & 0.205 & 0.682 \\
& & & & \\
A/California & & & & \\
Responders & 3 & $25.3(23.4)$ & $9.5(13.7)$ & $0.38(0.45)$ \\
Non-responders & 11 & $34(23)$ & $15.5(25.2)$ & $1.21(1.58)$ \\
p Value & & 0.694 & 0.640 & 0.413 \\
\hline
\end{tabular}

Values are mean (SD). 
unchanged, while decreases in IgM, IgE, anti-dsDNA and antiC1q antibodies were seen.

As far as we know, the immune response to active immunisation of patients with RA treated with rituximab has not been studied before. We now provide evidence of a humoral response to influenza virus vaccine by some of our rituximabtreated patients. The observed significantly decreased response might be attributed to a decrease in the amount of B cells after rituximab treatment or to an increase of regulatory $T$ cells. Indeed, previous studies showed a slow and delayed repopulation of CD27+ memory B cells with a significant reduction of their level (to $<50 \%$ ) for more than 2 years. ${ }^{20}$ On the other hand, a substantial proportion of the patients treated with rituximab did respond to the vaccine despite total B-cell depletion. This might be explained by the presence of plasma cells, which originate from marginal zone B cells less sensitive to rituximab treatment, that continue to secrete antibodies for extended periods of time. ${ }^{21}$ Another possible explanation is that early differentiating B cells with low-level expression of CD20 "survived" and became the source for the antibodies that had been produced.

In conclusion, this study has confirmed that vaccination against influenza is safe and the findings showed that it generated an appreciable humoral response in patients with RA treated with rituximab, although this response was lower than that seen in non-rituximab-treated patients with RA or in healthy controls. We are aware of the limitations of this study, which include a relatively small number of treated patients and controls. Based on our present data, however, we feel that vaccination against influenza, which is strongly indicated in RA, can also be safely recommended for patients who are being treated with rituximab.

Competing interests: None declared.

Ethics approval: Approved by the ethics committees of Tel Aviv Sourasky Medical Centre and the Rambam Health Care Centre.

\section{REFERENCES}

1. Gabriel SE, Crowson CS, Kremers NM, Doran MF, Turesson C, O'Fallon WM, et al. Mortality in rheumatoid arthritis: have we made impacts in 4 decades? Arthritis Rheum 2003;48:54-8.

2. Symmons DP, Jones MA, Scott DL. Long-term mortality outcome in patients with rheumatoid arthritis: early presenters continue to do well. J Rheumatol 1998;25:1072-7.
3. Doran MF, Crowson CS, Pond GR, O'Fallon WM, Gabriel SE. Frequency of infection in patients with rheumatoid arthritis compared with controls. Arthritis Rheum 2002; 46:2287-93.

4. Doran MF, Crowson CS, Pond GR, O'Fallon WM, Gabriel SE. Predictors of infection in rheumatoid arthritis. Arthritis Rheum 2002;46:2294-300.

5. Smith NM, Bresee JS, Shay DK, Uyeki TM, Cox NJ, Strikas RA. Prevention and control of influenza: Recommendation of the Advisory Committee on Immunization Practices (ACIP). National Center for Immunization and Respiratory Diseases (proposed). MMWR Recomm Rep 2006;55(RR-10):1-42.

6. Fomin I, Caspi D, Levy V, Varsano N, Shalev Y, Paran D, et al. Vaccination against influenza in rheumatoid arthritis: the effect of disease modifying drugs, including TNF alpha blockers. Ann Rheum Dis 2006;65:191-4.

7. Chalmers A, Scheifele D, Patterson C, Williams D, Weber J, Shuckett R, et al. Immunization of patients with rheumatoid arthritis against influenza: a study of vaccine safety and immunogenicity. J Rheumatol 1994:21:1203-6.

8. Edwards JC, Szczepanski L, Szechinski J, Filipowicz-Sosnowska A, Emery P, Close $\mathrm{DR}$, et al. Efficacy of B-cell-targeted therapy with rituximab in patients with rheumatoid arthritis. N Engl J Med 2004;350:2572-81.

9. Cambridge G, Leandro MJ, Teodorescu M, Manson J, Rahman A, Isenberg DA, et al. B cell depletion therapy in systemic lupus erythematosus: effect on autoantibody and antimicrobial antibody profiles. Arthritis Rheum 2006:54:3612-22.

10. Arnett FC, Edworthy SM, Bloch DA, McShane DJ, Fries JF, Cooper NS, et al. The American Rheumatism Association 1987 revised criteria for the classification of rheumatoid arthritis. Arthritis Rheum 1988;31:315-24.

11. Committee for Proprietary Medicinal Products (CPMC). Note for guidance on harmonization of requirements for influenza vaccines. CPMB/BWP/214/96, 1996 Circular No 96-0666;1-22.

12. Committee for Proprietary Medicinal Products (CPMC). Concept paper on the revision of the CPMP/BWP note for guidance on harmonization of requirements for influenza vaccines, 2001, CPMP/EPW/1045/01

13. Palmer DF, Coleman MT, Dowdle WR, Schild DC. Advanced laboratory techniques for influenza diagnosis. Public Health Service. US Department of Health, Education and Welfare. Atlanta: Centers for Disease Control, 1975.

14. Horwitz SM, Negrin RS, Blume KG, Breslin S, Stuart MJ, Stockerl-Goldstein KE, et al. Rituximab as adjuvant to high-dose therapy and autologous hematopoietic stem cell transplantation for aggressive non-Hodgkin lymphoma. Blood 2004;103:777-83.

15. Van der Kolk LE, Baars JW, Prins MH, van Oers MH. Rituximab treatment results in impaired secondary humoral immune responsiveness. Blood 2002;100:2257-9.

16. Bearden CM, Agarwal A, Book BK, Vieira CA, Sidner RA, Ochs HD, et al. Rituximab inhibits the in vivo primary and secondary antibody response to a neoantigen, bacteriophage phiX174. Am J Transplant 2005:5:50-7.

17. Dorner T, Radbruch A. Selecting B cells and plasma cells to memory. J Exp Med 2005:201:497-9.

18. Cambridge G, Stohl W, Leandro MJ, Migone TS, Hilbert DM, Edwards J. Circulating levels of B lymphocyte stimulator in patients with rheumatoid arthritis following rituximab treatment: relationships with B cell depletion, circulating antibodies, and clinical relapse. Arthritis Rheum 2006;54:723-32.

19. Vallerskog T, Gunnarsson I, Widhe M, Risselada A, Klareskog L, Vollenhoven R, et al. Treatment with rituximab affects both the cellular and the humoral arm of the immune system in patients with SLE. Clin Immunol 2007;122:62-74.

20. Roll P, Palanichamy A, Kneitz C, Dorner T, Tony HP. Regeneration of B cell subsets after transient B cell depletion using anti-CD20 antibodies in rheumatoid arthritis. Arthritis Rheum 2006:54:2377-86.

21. Slifka MK, Antia R, Whitmire JK, Ahmed R. Humoral immunity due to long-lived plasma cells. Immunity 1998;8:363-72. 